\title{
RENDIMIENTO Y CALIDAD DE LA MORERA (Morus alba) FERTILIZADA CON NITRÓGENO, FÓSFORO Y POTASIO ${ }^{1}$
}

\author{
Carlos Boschini-Figueroa ${ }^{2}$, Claudio Fabián Vargas-Rodríguez ${ }^{2}$
}

\begin{abstract}
RESUMEN
Rendimiento y calidad de la morera (Morus alba) fertilizada con nitrógeno, fósforo y potasio. El objetivo del presente trabajo fue determinar el efecto del nitrógeno, fósforo y potasio, aplicados al suelo, sobre el rendimiento y calidad bromatológica de la morera, a $1.542 \mathrm{msnm}$ en una plantación de morera establecida en el 2002, con una población de 25.000 plantas por hectárea en Costa Rica. Se efectuó una poda en mayo del 2007 a $60 \mathrm{~cm}$ sobre el nivel del suelo y se cosechó a los 84 días de rebrote. El rendimiento anual de materia seca mostró diferencias $(\mathrm{p}<0,05)$ entre los niveles de nitrógeno, fósforo y potasio aplicados. La mayor concentración de materia seca se produjo con $112,5 \mathrm{~kg}$ de $\mathrm{N} / \mathrm{ha} / \mathrm{año}, 30 \mathrm{~kg}$ de $\mathrm{P} / \mathrm{ha} / \mathrm{año} \mathrm{y} 225 \mathrm{~kg}$ de $\mathrm{K} / \mathrm{ha} / \mathrm{año}$. El porcentaje más alto de proteína cruda se obtuvo con $112,5 \mathrm{~kg}$ de $\mathrm{N} / \mathrm{ha} /$ año ( $\mathrm{p}>0,05)$, la respuesta fue similar con diferentes cantidades de fósforo y potasio. La concentración de fibra neutro detergente se afectó $(\mathrm{p}<0,05)$ conforme aumentaron las cantidades de nitrógeno, y también mostró variaciones marcadas $(\mathrm{p}<0,01)$ con la aplicación de fósforo y potasio. Los diferentes niveles de NPK afectaron poco $(p>0,05)$ la fibra ácido detergente; sin embargo, se observó que al incrementar el nitrógeno la hemicelulosa decreció $(\mathrm{p}<0,05)$ y aumentó la celulosa al aumentar el nitrógeno y fósforo, mientras decreció $(\mathrm{p}<0,05)$ con altos niveles de potasio. La producción de materia seca resultó altamente significativa $(\mathrm{p}<0,01)$ a la aplicación conjunta de nitrógeno, fósforo y potasio, no así los contenidos de proteína cruda y de fibra neutro detergente $(\mathrm{p}>0,05)$.
\end{abstract}

Palabras claves: Nutrición animal, arbusto, forraje, fertilización, NPK.

\begin{abstract}
Yield and quality of mulberry (Morus alba) fertilized with nitrogen, phosphorus and potassium. The objective of this work was to determine the effect of nitrogen, phosphorus and potassium applied to the soil, on yield and bromatological quality of mulberry. The experiment was conducted in Costa Rica at 1542 masl, in a mulberry plantation with a population of 25000 plants/ha established in 2002. The plants were homogenized by prunning at $60 \mathrm{~cm}$ above the soil surface and 84 days after were harvested for the evaluation. The annual mulberry dry matter yield showed important differences between applied levels of nitrogen, phosphorus and potassium $(\mathrm{p}<0.05)$. The highest concentration of dry matter was observed with $112.5 \mathrm{~kg}$ of N/ha/year, $30 \mathrm{~kg}$ of P/ha/year and $225 \mathrm{~kg} \mathrm{~K} / \mathrm{ha} /$ year. The highest percentage of crude protein was obtained with $112.5 \mathrm{~kg}$ of N/ha/year but with similar results $(\mathrm{p}>0.05)$ were observed when different amounts of phosphorus and potassium were applied. The concentration of neutral detergent fiber was affected $(\mathrm{p}<0.05)$ when the levels of nitrogen were increased and considerable variations were observed with addition of phosphorus and potassium. The different levels of NPK did not show important effects on acid detergent fiber; however, as the nitrogen levels increased the hemicelulose decreased $(\mathrm{p}<0.05)$, and the cellulose augmented with high levels of nitrogen and phosphorus, but decreased significantly with elevated amounts of potassium. The production of dry matter resulted highly significant $(\mathrm{p}<0.01)$ when nitrogen, phosphorus and potassium where applied together, but crude protein and neutral detergent fiber did not show the same pattern $(\mathrm{p}>0.05)$.
\end{abstract}

Key words: Animal nutrition, shrub, forage, fertilization, NPK.

\footnotetext{
1 Recibido: 23 de marzo, 2009. Aceptado: 16 de noviembre, 2009. Inscrito en la Vicerrectoría de Investigación, proyecto No 737-A4-134. Universidad de Costa Rica. San José, Costa Rica.

2 Estación Experimental Alfredo Volio Mata, Facultad de Ciencias Agroalimentarias, Universidad de Costa Rica. Cartago, Costa Rica. carlos. boschini@ucr.ac.cr; fabian.vargas@ucr.ac.cr
} 


\section{INTRODUCCIÓN}

La morera se ha venido usando como fuente forrajera en la alimentación de bovinos y caprinos (Rodríguez et al. 1994, González 1996). Es una especie arbórea que en condiciones de crecimiento libre alcanza 12 metros de altura; sin embargo, se ha adaptado de manera excelente en Mesoamérica para la producción de follaje de corte, cosechada a intervalos periódicos y sucesivos a través del año (Benavides et al. 1986, Benavides 1981). En esta modalidad se comporta como una planta arbustiva perenne, de rápido crecimiento con rebrotes foliares vigorosos y un sistema radical fuerte y profuso (Paolieri 1970, Tieng-Zing et al. 1988).

La producción de biomasa en explotación intensiva, sembrada en suelos bien drenados, zonas de alta luminosidad y con una precipitación media o alta, muestra en Costa Rica rendimientos de biomasa fresca de 70 - 119 t/ha/año (Boschini et al. 1998, Espinoza, 1996). Se ha observado una buena respuesta a la fertilización nitrogenada química (Rodríguez et al. 1994), así como con el compostaje (Elizondo 2007). En Centroamérica, se han reportado rendimientos de 19 a $28 \mathrm{t} / \mathrm{ha} / \mathrm{año}$ de materia seca con plantas enteras, cosechadas a $60-80 \mathrm{~cm}$ sobre el nivel del suelo, en intervalos de poda de seis a 12 semanas y con densidades de siembra de 20000 plantas por hectárea (Blanco 1992, Rodríguez et al. 1994). Los resultados de los experimentos en Mesoamérica muestran una tendencia a que el rendimiento por área mejora al aumentar la densidad de siembra y el plazo entre podas (Boschini et al. 1998, Boschini et al. 1999). La producción foliar es mayor a la de tallo durante los primeros 100 días de rebrote. La calidad bromatológica de las hojas, particularmente el contenido de proteína, se mantiene constante durante los primeros cuatro a cinco meses y posteriormente decrece conforme predomina la producción de tallo (Estrada 1995, Benavides 1986, Benavides et al. 1994, Velazquez et al. 1992).

Los grandes rendimientos de biomasa por unidad de área y altos contenidos de proteína en las hojas como en la fracción comestible de los tallos, indican la necesidad de reponer al suelo el nitrógeno extraído por la planta, así como los elementos de fósforo y potasio. En suelos de origen volcánico la IFA (Asociación Internacional de Industriales de Fertilizantes) recomiendan 300, 160 y $200 \mathrm{~kg}$ /ha/año de $\mathrm{N}, \mathrm{P}_{2} \mathrm{O}_{5}$ y $\mathrm{K}_{2} \mathrm{O}$, respectivamente (IFA 1992). Rodríguez et al. (1994) aplicaron dosis de 0, 40 y $80 \mathrm{~kg}$ de N/ha/corte en Guatemala, con rendimientos aceptables, pero observaron una baja respuesta en el incremento de proteína cruda en las hojas (17,5 a $18 \%$ ). Benavides et al. (1994) emplearon niveles de fertilización nitrogenada entre 0 a $480 \mathrm{~kg} / \mathrm{ha} /$ año en Costa Rica, durante tres años, obteniendo rendimientos promedios de 19 a 30 toneladas de materia seca por ha/año. En condiciones tropicales, la morera ha mostrado altos rendimientos de materia seca a través de los ciclos anuales de producción (Boschini 2002) y dada esa productividad, con frecuencia se presume que es una especie altamente extractiva y demandante de nutrientes del suelo. Se ha observado que la morera en cultivos de alta densidad de siembra mantiene altos volúmenes de biomasa, pero la calidad bromatológica decrece considerablemente. El objetivo del presente trabajo fue determinar el efecto del nitrógeno, fósforo y potasio, aplicados al suelo, sobre el rendimiento y calidad bromatológica de la morera.

\section{MATERIALES Y MÉTODOS}

El trabajo de campo se llevó a cabo en la Estación Experimental Alfredo Volio Mata, Universidad de Costa Rica, Cartago, Costa Rica, ubicada a $10^{\circ}$ latitud norte y $84^{\circ}$ longitud oeste, a $1.542 \mathrm{msnm}$ de altitud, con una precipitación media anual de $2.050 \mathrm{~mm}$, distribuida entre los meses de mayo y noviembre. En los restantes meses se encuentra el periodo seco. La temperatura media es de $19,5^{\circ} \mathrm{C}$ y una humedad relativa promedio de $84 \%$. El suelo está clasificado como Typic Distrandepts, de origen volcánico (Vásquez 1982), caracterizado por tener una profundidad media con buen drenaje natural, una fertilidad media en los primeros $30 \mathrm{~cm}$ (un pH de 6,1, 7,5\% de materia orgánica, 0,48\% de nitrógeno total, $12 \mathrm{mg} / 1$ de fósforo, $1,03 \mathrm{cmol} / 1$ de potasio, $8 \mathrm{cmol} / 1$ de calcio y $2,7 \mathrm{cmol} / 1$ de magnesio, $12 \mathrm{mg} / \mathrm{l}$ de cobre, 2,6 mg/l de zinc, 2,3 $\mathrm{mg} / \mathrm{l}$ de manganeso y $110 \mathrm{mg} / \mathrm{l}$ de hierro). La zona se tipifica como bosque húmedo montano (Bhb) bajo dentro de la clasificación ecológica (Vásquez 1982).

Se usó una plantación de $7.000 \mathrm{~m}^{2}$, establecida en mayo de 2002, con distancias entre surcos de $1 \mathrm{~m}$ por $40 \mathrm{~cm}$ entre plantas, equivalente a 25.000 plantas por hectárea. En mayo del 2007, se efectuó una poda de uniformización a $60 \mathrm{~cm}$ de altura sobre el nivel del suelo. La aplicación de los tratamientos fertilizantes se 
programó 15 días posteriores a esa fecha, cuando los rebrotes alcanzaron $2,5 \mathrm{~cm}$ de largo.

El lote fue dividido en 76 parcelas experimentales para distribuir los tratamientos, dejando dos metros de separación alrededor de cada una de ellas. Se empleó un diseño de bloques al azar con cuatro repeticiones y tratamientos en un arreglo factorial de compuesto central y rotable $5^{3}$ (Cochran y Cox 1957, Boschini 2008). El modelo estadístico empleado fue el siguiente:

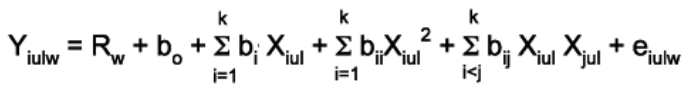

donde:

$\mathrm{Y}_{\text {iulw }}=$ Observación l-ésima en w-ésimo bloque en el u-ésimo nivel del i-ésimo factor.

$\mathrm{R}_{\mathrm{w}}=$ Bloque u-ésimo.

$\mathrm{b}_{\mathrm{o}} \quad=$ Valor de la intersección en la superficie de respuesta.

$b_{i} \quad=$ Pendiente lineal del i-ésimo factor.

$\mathrm{b}_{\mathrm{ii}} \quad=$ Pendiente cuadrática del i-ésimo factor.

$\mathrm{b}_{\mathrm{ij}} \quad=$ Pendiente de la interacción del i-ésimo factor $\mathrm{x}$ el j-ésimo factor.
$\mathrm{X}_{\mathrm{iul}}=$ Observación l-ésima en el nivel u-ésimo del i-ésimo factor.

$\mathrm{X}_{\mathrm{jul}} \quad=$ Observación l-ésima en el nivel u-ésimo del j-ésimo factor.

$\mathrm{e}_{\mathrm{iulw}}=$ Error experimental en la observación l-ésima en el w-ésimo bloque en el u-ésimo nivel del i-ésimo factor.

Las variables estudiadas fueron; nitrógeno, fósforo y potasio, en los siguientes niveles: $35,112,5,225$, 337,5 y 414,2 kg/ha/año; 9,55, 30, 60, 90 y 110,45 $\mathrm{kg} / \mathrm{ha} /$ año y $23,87,75,150,225$ y $276,13 \mathrm{~kg} / \mathrm{ha} / \mathrm{año}$, en el respectivo orden de los factores descritos. La combinación del tratamiento central tuvo 20 repeticiones y en los restantes sólo cuatro. La cantidad de fertilizante en cada tratamiento abarcó el periodo experimental de tres meses, aplicado en forma individual y uniforme en cada parcela experimental. En el Cuadro 1 se muestra los tratamientos experimentales y las cantidades de nitrato de amonio $(33 \% \mathrm{~N})$, triple superfosfato $(46 \% \mathrm{P})$ y cloruro de potasio $(60 \% \mathrm{~K})$ empleados en la mezcla física.

Cada parcela fue cosechada completamente al concluir el periodo experimental de 84 días (12

Cuadro 1. Tratamientos experimentales y materias primas empleadas en la mezcla física de fertilizante aplicado en morera. Cartago, Costa Rica. 2007.

\begin{tabular}{|c|c|c|c|c|c|c|c|c|}
\hline \multirow{2}{*}{$\begin{array}{l}\text { Trata- } \\
\text { mientos }\end{array}$} & \multirow{2}{*}{$\begin{array}{l}\text { Tratamientos } \\
\text { codificados }\end{array}$} & \multirow[t]{2}{*}{ Rep } & \multicolumn{3}{|c|}{ Cantidades en $\mathrm{kg} / \mathrm{ha} / \mathbf{a n ̃ o}$} & \multicolumn{3}{|c|}{ Cantidades en $\mathrm{kg} / \mathrm{ha} / \mathbf{a n ̃ o}$} \\
\hline & & & $\mathbf{N}$ & $\mathbf{P}$ & $\mathbf{K}$ & $\begin{array}{c}\text { Nitrato } \\
\text { de amonio }\end{array}$ & $\begin{array}{c}\text { Triple } \\
\text { superfosfato }\end{array}$ & $\begin{array}{c}\text { Cloruro } \\
\text { potasio }\end{array}$ \\
\hline $\mathrm{N}_{1} \mathrm{P}_{3} \mathrm{~K}_{3}$ & $(-1,68 ; 0 ; 0)$ & 4 & 35,80 & 60,00 & 150,00 & 108,48 & 130,43 & 250,00 \\
\hline $\mathrm{N}_{2} \mathrm{P}_{2} \mathrm{~K}_{2}$ & $(-1 ;-1 ;-1)$ & 4 & 112,50 & 30,00 & 75,00 & 340,91 & 65,22 & 125,00 \\
\hline $\mathrm{N}_{2} \mathrm{P}_{2} \mathrm{~K}_{4}$ & $(-1 ;-1 ; 1)$ & 4 & 112,50 & 30,00 & 225,00 & 340,91 & 65,22 & 375,00 \\
\hline $\mathrm{N}_{2} \mathrm{P} 4 \mathrm{~K}_{2}$ & $(-1 ; 1 ;-1)$ & 4 & 112,50 & 90,00 & 75,00 & 340,91 & 195,65 & 125,00 \\
\hline $\mathrm{N}_{2} \mathrm{P}_{4} \mathrm{~K}_{4}$ & $(-1 ; 1 ; 1)$ & 4 & 112,50 & 90,00 & 225,00 & 340,91 & 195,65 & 375,00 \\
\hline $\mathrm{N}_{3} \mathrm{P}_{1} \mathrm{~K}_{3}$ & $(0 ;-1,68 ; 0)$ & 4 & 225,00 & 9,55 & 150,00 & 681,82 & 20,76 & 250,00 \\
\hline $\mathrm{N}_{3} \mathrm{P}_{3} \mathrm{~K}_{1}$ & $(0 ; 0 ;-1,68)$ & 4 & 225,00 & 60,00 & 23,87 & 681,82 & 130,43 & 39,78 \\
\hline $\mathrm{N}_{3} \mathrm{P}_{3} \mathrm{~K}_{3}$ & $(0 ; 0 ; 0)$ & 20 & 225,00 & 60,00 & 150,00 & 681,82 & 130,43 & 250,00 \\
\hline $\mathrm{N}_{3} \mathrm{P}_{3} \mathrm{~K}_{5}$ & $(0 ; 0 ; 1,68)$ & 4 & 225,00 & 60,00 & 276,13 & 681,82 & 130,43 & 460,22 \\
\hline $\mathrm{N}_{3} \mathrm{P}_{5} \mathrm{~K}_{3}$ & $(0 ; 1,68 ; 0)$ & 4 & 225,00 & 110,45 & 150,00 & 681,82 & 240,11 & 250,00 \\
\hline $\mathrm{N}_{4} \mathrm{P}_{2} \mathrm{~K}_{2}$ & $(1 ;-1 ;-1)$ & 4 & 337,50 & 30,00 & 75,00 & $1.022,73$ & 65,22 & 125,00 \\
\hline $\mathrm{N}_{4} \mathrm{P}_{2} \mathrm{~K}_{4}$ & $(1 ;-1 ; 1)$ & 4 & 337,50 & 30,00 & 225,00 & $1.022,73$ & 65,22 & 375,00 \\
\hline $\mathrm{N}_{4} \mathrm{P}_{4} \mathrm{~K}_{2}$ & $(1 ; 1 ;-1)$ & 4 & 337,50 & 90,00 & 75,00 & $1.022,73$ & 195,65 & 125,00 \\
\hline $\mathrm{N}_{4} \mathrm{P}_{4} \mathrm{~K}_{4}$ & $(1 ; 1 ; 1)$ & 4 & 337,50 & 90,00 & 225,00 & $1.022,73$ & 195,65 & 375,00 \\
\hline $\mathrm{N}_{5} \mathrm{P}_{3} \mathrm{~K}_{3}$ & $(1,68 ; 0 ; 0)$ & 4 & 414,20 & 60,00 & 150,00 & $1.255,15$ & 130,43 & 250,00 \\
\hline
\end{tabular}


semanas), cortando las plantas a $60 \mathrm{~cm}$ sobre el nivel de suelo. La producción de biomasa fresca se pesó en el campo. Posteriormente se obtuvo una muestra compuesta del material cosechado. Cada una fue pesada en fresco, secada a $60{ }^{\circ} \mathrm{C}$ durante 48 horas hasta alcanzar un peso constante y pesada nuevamente en seco. Estas muestras se molieron en un molino Willey, con malla de $1 \mathrm{~mm}$ de paso. Posteriormente, se determinó el contenido de materia seca a $105{ }^{\circ} \mathrm{C}$ (AOAC 2002), proteína cruda por el método micro Kjeldahl (AOAC 2002), la fibra neutro detergente y sus fracciones (fibra ácido detergente, hemicelulosa, celulosa y lignina) mediante la técnica de Goering y Van Soest (1970), el extracto etéreo y el contenido de cenizas totales (AOAC 2002). Se estimaron los rendimientos de biomasa seca en planta entera a partir de los muestreos realizados. Los resultados se analizaron con el PROC GLM del paquete estadístico SAS (1985) con el modelo propuesto. A todas las variables que resultaron significativas $(\mathrm{P}<0,05)$ en el análisis de varianza, se les hizo un análisis de regresión polinomial y posteriormente se determinó mediante derivadas parciales los puntos de máxima o mínima respuesta de cada factor de fertilizante aplicado.

\section{RESULTADOS Y DISCUSIÓN}

La producción de materia fresca y seca medida en cada nivel de fertilización nitrogenada, fosfórica y potásica se presentan en el Cuadro 2. Se observa que la producción de materia seca incrementó significativamente $(\mathrm{P}<0,05)$ un $34,1 \%$ al pasar del nivel de 35 a $112,5 \mathrm{~kg}$ de $\mathrm{N} / \mathrm{ha} / \mathrm{año}$ y posteriormente decreció un $16,8 \%$ al incrementar a $225 \mathrm{~kg}$ de N/ha/año ( $>>0,05)$. Con cantidades mayores, la cosecha de materia seca tiende a mantenerse. Esta producción se vió significativamente afectada $(\mathrm{P}<0,05)$ en un $30,5 \%$ al aumentar el fósforo de 9,55 a $30 \mathrm{~kg} / \mathrm{ha} / \mathrm{año}$. Con cantidades mayores no se observan cambios importantes $(\mathrm{P}>0,05)$, con un leve repunte cuando se usaron $90 \mathrm{~kg}$ de $\mathrm{P} / \mathrm{ha} /$ año y un descenso prominente $(\mathrm{P}<0,05)$ en el nivel

Cuadro 2. Promedios de producción de biomasa y composición nutricional de la morera en cada fuente de tratamiento. Cartago, Costa Rica. 2007.

\begin{tabular}{|c|c|c|c|c|c|c|c|c|c|c|c|c|c|c|}
\hline \multicolumn{3}{|c|}{ Tratamientos } & \multicolumn{3}{|c|}{$\begin{array}{l}\text { Producción de } \\
\text { biomasa }\end{array}$} & \multicolumn{9}{|c|}{ Composición nutricional } \\
\hline $\mathbf{N}$ & $\mathbf{P}$ & $\mathbf{K}$ & & Materia & Materia & MS & PC & FND & FAD & HEMI & CEL & LIG & $\mathbf{E E}$ & CEN \\
\hline $\begin{array}{l}\text { kg/ha/ } \\
\text { año }\end{array}$ & $\begin{array}{c}\text { kg/ha/ } \\
\text { año }\end{array}$ & $\begin{array}{c}\text { kg/ha/ } \\
\text { año }\end{array}$ & $\mathbf{n}$ & $\begin{array}{c}\text { fresca, } \mathrm{kg} / \\
\text { ha/año }\end{array}$ & $\begin{array}{c}\text { seca, kg/ } \\
\text { ha/año }\end{array}$ & $\%$ & $\%$ & $\%$ & $\%$ & $\%$ & $\%$ & $\%$ & $\%$ & $\%$ \\
\hline 35,00 & & & 4 & 87.000 & 15.857 & 18,42 & 25,89 & 63,77 & 37,06 & 26,71 & 25,00 & 12,06 & 2,46 & 14,18 \\
\hline 112,50 & & & 16 & 111.675 & 21.263 & 18,85 & 20,34 & 59,89 & 36,51 & 22,34 & 21,52 & 16,03 & 1,85 & 14,32 \\
\hline 225,00 & & & 36 & 99.556 & 17.681 & 17,52 & 16,46 & 60,19 & 38,54 & 21,79 & 21,59 & 17,04 & 2,28 & 15,79 \\
\hline 337,50 & & & 16 & 96.000 & 17.183 & 17,67 & 15,64 & 59,23 & 37,89 & 21,34 & 23,14 & 16,48 & 2,28 & 15,40 \\
\hline 414,20 & & & 4 & 93.000 & 14.422 & 15,63 & 16,20 & 53,48 & 37,46 & 16,03 & 22,09 & 15,37 & 2,73 & 16,65 \\
\hline & 9,55 & & 4 & 115.000 & 23.385 & 20,37 & 15,96 & 59,39 & 37,51 & 21,89 & 20,99 & 16,52 & 2,45 & 14,58 \\
\hline & 30,00 & & 16 & 98.750 & 17.913 & 17,99 & 17,92 & 58,67 & 37,31 & 20,32 & 21,28 & 17,07 & 2,00 & 14,93 \\
\hline & 60,00 & & 36 & 97.333 & 16.820 & 17,12 & 17,43 & 58,96 & 38,42 & 20,81 & 21,92 & 16,58 & 2,31 & 15,74 \\
\hline & 90,00 & & 16 & 108.925 & 20.534 & 18,54 & 18,05 & 60,45 & 37,09 & 23,36 & 23,39 & 15,44 & 2,12 & 14,79 \\
\hline & 110,45 & & 4 & 85.000 & 14.646 & 17,29 & 17,34 & 68,97 & 38,11 & 29,62 & 23,05 & 15,06 & 2,49 & 16,79 \\
\hline & & 23,87 & 4 & 83.000 & 12.933 & 15,42 & 17,30 & 59,09 & 38,65 & 20,43 & 22,91 & 15,74 & 1,81 & 17,32 \\
\hline & & 75,00 & 16 & 101.500 & 18.373 & 17,89 & 18,00 & 59,94 & 38,88 & 21,06 & 24,07 & 16,54 & 2,10 & 15,10 \\
\hline & & 150,00 & 36 & 101.000 & 18.079 & 17,78 & 17,49 & 61,04 & 38,15 & 23,02 & 21,89 & 16,35 & 2,40 & 15,42 \\
\hline & & 225,00 & 16 & 106.175 & 20.074 & 18,63 & 17,98 & 59,18 & 35,52 & 22,62 & 20,60 & 15,97 & 2,03 & 14,62 \\
\hline & & 276,13 & 4 & 84.000 & 13.765 & 16,33 & 15,50 & 50,55 & 39,37 & 11,18 & 21,43 & 17,94 & 2,36 & 16,85 \\
\hline
\end{tabular}


de $110,45 \mathrm{~kg} / \mathrm{ha} /$ año. Similar respuesta significativa $(\mathrm{P}<0,05)$ se observa al aumentar las dosis de potasio. $\mathrm{Al}$ pasar de 23,87 a $75 \mathrm{~kg}$ de $\mathrm{K} / \mathrm{ha} / \mathrm{año}$, se produjo un aumento de $22,3 \%$ de biomasa seca, manteniéndose estable hasta el nivel de $225 \mathrm{~kg} / \mathrm{ha} / \mathrm{año}$. Posteriormente, decrece la producción $17,2 \%$ con la cantidad de $276,13 \mathrm{~kg} / \mathrm{ha} /$ año en forma significativa $(\mathrm{P}<0,05)$.

La mayor concentración de materia seca fue de $18,85 \%$ cuando se aplicaron $112,5 \mathrm{~kg}$ de N/ha/año, ligeramente inferior $(P>0,05)$ en el de $35 \mathrm{~kg} / \mathrm{ha} / \mathrm{año}$ y más baja $(\mathrm{P}<0,05)$ conforme aumentó la adición de nitrógeno al suelo. Entre los niveles de fósforo se observaron diferencias $(\mathrm{P}<0,01)$ sobre el porcentaje de materia seca. A $9,55 \mathrm{~kg} / \mathrm{ha} /$ año se manifiestó un 2,38\% más de materia seca que con un aporte de 30 $\mathrm{kg} / \mathrm{ha} / \mathrm{año}$. Esta diferencia se mantuvo también en los niveles superiores de fósforo aplicados al suelo. Las diferentes proporciones de potasio mostraron diferencias importantes $(\mathrm{P}<0,01)$ en el contenido de materia seca. El aporte más bajo y el más alto de potasio mostraron un decremento marcado de la concentración de materia seca, presentando en los rangos intermedios un valor estable entre 17,7 y $18,6 \%$ de materia seca.

El porcentaje de proteína cruda se afectó marcadamente $(\mathrm{P}<0,01)$ con la aplicación de nitrógeno. Se observó una gran diferencia $(\mathrm{P}<0,01)$ entre el nivel de 35 y $112,5 \mathrm{~kg} / \mathrm{ha} / \mathrm{año}$, y así entre ambos y los niveles superiores. No se encontró ninguna diferencia importante $(\mathrm{P}>0,05)$ con la aplicación de fósforo y potasio sobre el contenido de proteína cruda.

El contenido de fibra neutro detergente fue afectado $(\mathrm{P}<0,05)$ conforme aumentó el nivel de nitrógeno aplicado al suelo y también mostró variaciones muy marcadas $(\mathrm{P}<0,01)$ con la aplicación de fósforo y potasio. Valores altos de nitrógeno y de potasio $(414,2$ y $276,13 \mathrm{~kg} / \mathrm{ha} / \mathrm{año}$ ) disminuyeron la fibra neutra en $6 \% \mathrm{y}$ $9 \%$ respectivamente. Niveles altos de fósforo $(110,45$ $\mathrm{kg} / \mathrm{ha} / \mathrm{año}$ ) incrementaron el contenido de fibra neutro detergente en un $8 \%$ sobre los restantes niveles.

Al buscar cual de los componentes de la fibra neutra tuvo mayores variaciones, se observa que los diferentes niveles de nitrógeno, fósforo y potasio no produjeron diferencias importantes $(\mathrm{P}>0,05)$ en el contenido de fibra ácido detergente y por el contrario, tuvieron una marcada influencia $(\mathrm{P}<0,05)$ sobre el contenido de hemicelulosa. Conforme se aplicaron cantidades mayores de nitrógeno el contenido de hemicelulosa decreció entre 4 y $10 \%$, al aumentar el fósforo se incrementó entre 6 y $8 \%$ y altos niveles de potasio decrecieron entre 8 y $9 \%$ el contenido de hemicelulosa. La celulosa como parte de la fracción de fibra ácida no mostró diferencias importantes $(\mathrm{P}>0,05)$ entre los diferentes tratamientos de nitrógeno y fósforo, y con niveles altos de potasio $(276,13 \mathrm{~kg} / \mathrm{ha} / \mathrm{año})$ se observó una disminución importante $(\mathrm{P}<0,05)$ de 11-12\%. El otro componente de la fibra ácida que es la lignina, mostró variaciones importantes $(\mathrm{P}<0,05)$ entre los niveles de nitrógeno, pero no se apreciaron diferencias significativas $(\mathrm{P}>0,05)$ con la aplicación de los niveles de fósforo y potasio.

El extracto etéreo mostró variaciones importantes $(\mathrm{P}<0,01)$ entre los diferentes niveles de nitrógeno, no así entre los de fósforo y potasio $(\mathrm{P}>0,05)$. La mayor diferencia de extracto etéreo observada se dio entre 112,5 y $414,2 \mathrm{~kg}$ de N/ha/año.

El contenido de cenizas totales varió entre 2 y 2,5 $\%$ entre los diferentes niveles de nitrógeno $(\mathrm{P}<0,01)$, fósforo $(\mathrm{P}<0,05)$ y potasio $(\mathrm{P}<0,01)$ en forma significativa, mostrando un patrón de aumento lineal en la concentración de cenizas al incrementar las cantidades de nitrógeno y fósforo y un patrón cuadrático de rendimientos decrecientes al aumentar los niveles de potasio.

En el Cuadro 3 se muestran los valores de producción de biomasa fresca y seca y la composición nutricional de la morera bajo el efecto de dos factores fertilizantes: nitrógeno y fósforo, nitrógeno y potasio, así como fósforo y potasio. Tanto en materia verde como materia seca se observaron diferencias significativas $(\mathrm{P}<0,01)$ en la interacción del nitrógeno con el fósforo, no así entre nitrógeno y potasio, ni entre fósforo y potasio $(\mathrm{P}>0,05)$.

Las interacciones de nitrógeno $\mathrm{x}$ fósforo $\mathrm{y}$ de nitrógeno $\mathrm{x}$ potasio sobre el contenido de materia seca fue altamente significativa $(\mathrm{P}<0,01)$, mientras entre el fósforo y el potasio la interacción no mostró importancia $(\mathrm{P}>0,05)$. Las interacciones nitrógeno $\mathrm{x}$ fósforo, nitrógeno $\mathrm{x}$ potasio $\mathrm{y}$ fósforo $\mathrm{x}$ potasio no presentaron ningún efecto importante sobre el contenido de proteína y fibra neutro detergente, tampoco en ninguno de los componentes de la pared celular (fibra ácido detergente, hemicelulosa, celulosa y lignina). El efecto de interacción de nitrógeno $\mathrm{x}$ fósforo fue altamente significativo $(\mathrm{P}<0,01)$ sobre el contenido de extracto etéreo y de las cenizas totales. Las otras interacciones no mostraron un efecto importante $(\mathrm{P}>0,05)$ sobre estos dos componentes de la materia seca. 
Cuadro 3. Promedios de producción de biomasa y composición nutricional de la morera, en los tratamientos agrupados en dos fuentes. Cartago, Costa Rica. 2007.

\begin{tabular}{|c|c|c|c|c|c|c|c|c|c|c|c|c|c|c|}
\hline \multicolumn{3}{|c|}{ Tratamientos } & & \multicolumn{2}{|c|}{$\begin{array}{l}\text { Producción de } \\
\text { biomasa }\end{array}$} & \multicolumn{9}{|c|}{ Composición nutricional } \\
\hline $\mathbf{N}$ & $\mathbf{P}$ & $\mathbf{K}$ & & Materia & Materia & MS & PC & FND & FAD & HEMI & CEL & LIG & $\mathbf{E E}$ & CEN \\
\hline $\begin{array}{c}\text { kg/ha/ } \\
\text { año }\end{array}$ & $\begin{array}{c}\text { kg/ha/ } \\
\text { año }\end{array}$ & $\begin{array}{c}\text { kg/ha/ } \\
\text { año }\end{array}$ & n & $\begin{array}{c}\text { fresca, kg/ } \\
\text { ha/año }\end{array}$ & $\begin{array}{c}\text { seca, kg/ } \\
\text { ha/año }\end{array}$ & $\%$ & $\%$ & $\%$ & $\%$ & $\%$ & $\%$ & $\%$ & $\%$ & $\%$ \\
\hline 35,00 & 60,00 & & 4 & 87.000 & 15857 & 18,42 & 25,89 & 63,77 & 37,06 & 26,71 & 25,00 & 12,06 & 2,46 & 14,18 \\
\hline 112,50 & 30,00 & & 8 & 92.500 & 16868 & 18,15 & 20,27 & 59,24 & 36,84 & 20,30 & 21,32 & 17,61 & 1,98 & 14,97 \\
\hline 112,50 & 90,00 & & 8 & 130.850 & 25.658 & 19,55 & 20,41 & 60,54 & 36,18 & 24,37 & 21,73 & 14,45 & 1,71 & 13,67 \\
\hline 225,00 & 9,55 & & 4 & 115.000 & 23.385 & 20,37 & 15,96 & 59,39 & 37,51 & 21,89 & 20,99 & 16,52 & 2,45 & 14,58 \\
\hline 225,00 & 60,00 & & 28 & 99.429 & 17.300 & 17,15 & 16,40 & 59,05 & 38,75 & 20,65 & 21,46 & 17,40 & 2,23 & 15,83 \\
\hline 225,00 & 110,45 & & 4 & 85.000 & 14.646 & 17,29 & 17,34 & 68,97 & 38,11 & 29,62 & 23,05 & 15,06 & 2,49 & 16,79 \\
\hline 337,50 & 30,00 & & 8 & 105.000 & 18.957 & 17,82 & 15,58 & 58,10 & 37,78 & 20,33 & 21,25 & 16,53 & 2,02 & 14,88 \\
\hline 337,50 & 90,00 & & 8 & 87.000 & 15.409 & 17,52 & 15,69 & 60,36 & 38,01 & 22,36 & 25,04 & 16,43 & 2,53 & 15,91 \\
\hline 414,20 & 60,00 & & 4 & 93.000 & 14.422 & 15,63 & 16,20 & 53,48 & 37,46 & 16,03 & 22,09 & 15,37 & 2,73 & 16,65 \\
\hline 35,00 & & 150,00 & 4 & 87.000 & 15.857 & 18,42 & 25,89 & 63,77 & 37,06 & 26,71 & 25,00 & 12,06 & 2,46 & 14,18 \\
\hline 112,50 & & 75,00 & 8 & 109.500 & 20.828 & 18,95 & 20,51 & 60,34 & 38,56 & 21,78 & 23,33 & 15,23 & 1,82 & 14,26 \\
\hline 112,50 & & 225,00 & 8 & 113.850 & 21.699 & 18,75 & 20,16 & 59,43 & 34,45 & 22,89 & 19,72 & 16,82 & 1,87 & 14,38 \\
\hline 225,00 & & 23,87 & 4 & 83.000 & 12.933 & 15,42 & 17,30 & 59,09 & 38,65 & 20,43 & 22,91 & 15,74 & 1,81 & 17,32 \\
\hline 225,00 & & 150,00 & 28 & 104.143 & 18.919 & 17,99 & 16,47 & 61,72 & 38,41 & 23,50 & 21,42 & 17,10 & 2,34 & 15,43 \\
\hline 225,00 & & 276,13 & 4 & 84.000 & 13.765 & 16,33 & 15,50 & 50,55 & 39,37 & 11,18 & 21,43 & 17,94 & 2,36 & 16,85 \\
\hline 337,50 & & 75,00 & 8 & 93.500 & 15.917 & 16,83 & 15,49 & 59,54 & 39,21 & 20,34 & 24,81 & 17,85 & 2,37 & 15,93 \\
\hline 337,50 & & 225,00 & 8 & 98.500 & 18.450 & 18,52 & 15,79 & 58,93 & 36,58 & 22,35 & 21,47 & 15,11 & 2,19 & 14,86 \\
\hline 414,20 & & 150,00 & 4 & 93.000 & 14.422 & 15,63 & 16,20 & 53,48 & 37,46 & 16,03 & 22,09 & 15,37 & 2,73 & 16,65 \\
\hline & 9,55 & 150,00 & 4 & 115.000 & 23.385 & 20,37 & 15,96 & 59,39 & 37,51 & 21,89 & 20,99 & 16,52 & 2,45 & 14,58 \\
\hline & 30,00 & 75,00 & 8 & 95.000 & 16.625 & 17,44 & 17,57 & 60,18 & 40,16 & 20,02 & 23,69 & 16,47 & 2,12 & 15,45 \\
\hline & 30,00 & 225,00 & 8 & 102.500 & 19.200 & 18,54 & 18,28 & 57,17 & 34,46 & 20,61 & 18,88 & 17,67 & 1,88 & 14,40 \\
\hline & 60,00 & 23,87 & 4 & 83.000 & 12.933 & 15,42 & 17,30 & 59,09 & 38,65 & 20,43 & 22,91 & 15,74 & 1,81 & 17,32 \\
\hline & 60,00 & 150,00 & 28 & 101.286 & 17.812 & 17,48 & 17,73 & 60,14 & 38,25 & 22,25 & 21,86 & 16,51 & 2,38 & 15,35 \\
\hline & 60,00 & 276,13 & 4 & 84.000 & 13.765 & 16,33 & 15,50 & 50,55 & 39,37 & 11,18 & 21,43 & 17,94 & 2,36 & 16,85 \\
\hline & 90,00 & 75,00 & 8 & 108.000 & 20.120 & 18,34 & 18,43 & 59,71 & 37,61 & 22,10 & 24,45 & 16,62 & 2,07 & 14,74 \\
\hline & 90,00 & 225,00 & 8 & 109.850 & 20.948 & 18,73 & 17,67 & 61,19 & 36,57 & 24,62 & 22,32 & 14,26 & 2,18 & 14,84 \\
\hline & 110,45 & 150,00 & 4 & 85000 & 14646 & 17,29 & 17,34 & 68,97 & 38,11 & 29,62 & 23,05 & 15,06 & 2,49 & 16,79 \\
\hline
\end{tabular}

En el Cuadro 4 se presentan los promedios de producción de biomasa y la composición nutricional de la materia seca bajo el efecto conjunto de los tres factores estudiados. La producción de materia verde y seca resultaron altamente significativos $(\mathrm{P}<0,01)$. En la Figura 1 se muestra la superficie de respuesta de la materia seca en los diferentes niveles de fósforo y potasio con seis dosis estandarizadas de nitrógeno. El contenido de proteína cruda y de pared celular no mostraron diferencias importantes $(\mathrm{P}>0,05)$ entre la variedad de niveles de las fuentes fertilizantes combinadas interactuantes. Tampoco se observó ningún efecto de interacción importante $(\mathrm{P}>0,05)$ entre los factores estudiados sobre los componentes de la pared celular (fibra ácido detergente, hemicelulosa, celulosa y lignina), el extracto etéreo y las cenizas totales.

En el Cuadro 5 se presentan las ecuaciones de predicción de la materia seca y de los componentes 
Cuadro 4. Promedios de producción de biomasa y composición nutricional de la morera, en los tratamientos estudiados. Cartago, Costa Rica. 2007.

\begin{tabular}{|c|c|c|c|c|c|c|c|c|c|c|c|c|c|c|}
\hline \multicolumn{4}{|c|}{ Tratamientos } & \multicolumn{2}{|c|}{$\begin{array}{l}\text { Producción de } \\
\text { biomasa }\end{array}$} & \multicolumn{9}{|c|}{ Composición nutricional } \\
\hline $\mathbf{N}$ & $\mathbf{P}$ & $\mathbf{K}$ & & Materia & Materia & MS & PC & FND & FAD & HEMI & CEL & LIG & $\mathbf{E E}$ & CEN \\
\hline $\begin{array}{l}\mathrm{kg} / \mathrm{ha} / \\
\text { año }\end{array}$ & $\begin{array}{l}\mathrm{kg} / \mathrm{ha} / \\
\text { año }\end{array}$ & $\begin{array}{l}\mathrm{kg} / \mathrm{ha} / \\
\text { año }\end{array}$ & $\mathbf{n}$ & $\begin{array}{c}\text { fresca, } \\
\text { kg/ha/año }\end{array}$ & $\begin{array}{c}\text { seca, kg/ } \\
\text { ha/año }\end{array}$ & $\%$ & $\%$ & $\%$ & $\%$ & $\%$ & $\%$ & $\%$ & $\%$ & $\%$ \\
\hline 35,00 & 60,00 & 150,00 & 4 & 87.000 & 15.857 & 18,42 & 25,89 & 63,77 & 37,06 & 26,71 & 25,00 & 12,06 & 2,46 & 14,18 \\
\hline 112,50 & 30,00 & 75,00 & 4 & 100.000 & 18.782 & 18,75 & 19,74 & 61,81 & 40,59 & 21,22 & 24,90 & 15,69 & 1,99 & 15,02 \\
\hline 112,50 & 30,00 & 225,00 & 4 & 85.000 & 14.955 & 17,56 & 20,80 & 56,67 & 33,10 & 19,39 & 17,74 & 19,53 & 1,98 & 14,92 \\
\hline 112,50 & 90,00 & 75,00 & 4 & 119.000 & 22.875 & 19,16 & 21,28 & 58,88 & 36,54 & 22,35 & 21,76 & 14,78 & 1,66 & 13,51 \\
\hline 112,50 & 90,00 & 225,00 & 4 & 142.700 & 28.442 & 19,95 & 19,53 & 62,19 & 35,81 & 26,39 & 21,70 & 14,12 & 1,77 & 13,83 \\
\hline 225,00 & 9,55 & 150,00 & 4 & 115.000 & 23.385 & 20,37 & 15,96 & 59,39 & 37,51 & 21,89 & 20,99 & 16,52 & 2,45 & 14,58 \\
\hline 225,00 & 60,00 & 23,87 & 4 & 83.000 & 12.933 & 15,42 & 17,30 & 59,09 & 38,65 & 20,43 & 22,91 & 15,74 & 1,81 & 17,32 \\
\hline 225,00 & 60,00 & 150,00 & 20 & 105.800 & 18.881 & 17,66 & 16,40 & 60,74 & 38,65 & 22,59 & 21,18 & 17,62 & 2,29 & 15,32 \\
\hline 225,00 & 60,00 & 276,13 & 4 & 84.000 & 13.765 & 16,33 & 15,50 & 50,55 & 39,37 & 11,18 & 21,43 & 17,94 & 2,36 & 16,85 \\
\hline 225,00 & 110,45 & 150,00 & 4 & 85.000 & 14.646 & 17,29 & 17,34 & 68,97 & 38,11 & 29,62 & 23,05 & 15,06 & 2,49 & 16,79 \\
\hline 337,50 & 30,00 & 75,00 & 4 & 90.000 & 14.469 & 16,13 & 15,40 & 58,55 & 39,73 & 18,82 & 22,48 & 17,25 & 2,25 & 15,89 \\
\hline 337,50 & 30,00 & 225,00 & 4 & 120.000 & 23.445 & 19,52 & 15,77 & 57,66 & 35,82 & 21,84 & 20,01 & 15,81 & 1,79 & 13,87 \\
\hline 337,50 & 90,00 & 75,00 & 4 & 97.000 & 17.365 & 17,53 & 15,58 & 60,54 & 38,68 & 21,85 & 27,15 & 18,46 & 2,49 & 15,97 \\
\hline 337,50 & 90,00 & 225,00 & 4 & 77.000 & 13.454 & 17,51 & 15,81 & 60,19 & 37,34 & 22,86 & 22,94 & 14,40 & 2,58 & 15,85 \\
\hline 414,20 & 60,00 & 150,00 & 4 & 93.000 & 14.422 & 15,63 & 16,20 & 53,48 & 37,46 & 16,03 & 22,09 & 15,37 & 2,73 & 16,65 \\
\hline 225 & 60 & 150 & & 100.353 & 18.063 & 17,78 & 17,58 & 59,76 & 37,84 & 21,77 & 22,11 & 16,36 & 2,22 & 15,36 \\
\hline
\end{tabular}

bromatológicos en función de los niveles de nitrógeno, fósforo y potasio aplicados en el experimento. Con ellas se estimaron los niveles máximos o mínimos de requerimientos de nitrógeno, fósforo y potasio por hectárea a aplicar anualmente (Cuadro 6) de acuerdo a la superficie de respuesta que logró el mayor ajuste. Tomando en cuenta todos los parámetros productivos y bromatológicos estudiados, el requerimiento promedio de nitrógeno fue $262 \mathrm{~kg} / \mathrm{ha}$ /año de fósforo 56,7 $\mathrm{kg} / \mathrm{ha}$ /año y de potasio $117,7 \mathrm{~kg} / \mathrm{ha} / \mathrm{año}$, equivalente a una proporción de 4,6:1:2 de NPK.

Los resultados experimentales presentados en el Cuadro 2, muestran rendimientos medios generales de morera en la planta entera muy similares a los reportados previamente en una plantación ubicada en la misma zona, con las similares prácticas de manejo (altura y frecuencia de corte) (Boschini et al. 1998). Es importante esta comparación para demostrar la persistencia de producción de esta planta al ser sometida a la defoliación periódica para la producción de forraje para nutrir bovinos y caprinos (Castro 1989, Boschini et al. 1999).

Los resultados muestran que la morera respondió en forma significativa $(\mathrm{P}<0,05)$ a la fertilización con nitrógeno, fósforo y potasio en forma individual como efectos principales. Espinoza (1996) con tres variedades de morera en diferentes zonas de Costa Rica observó una respuesta similar al aplicar diferentes niveles de nitrógeno (entre 180 y $540 \mathrm{~kg} / \mathrm{ha} / \mathrm{año}$ ). No se encontró en la literatura alguna observación de la respuesta del fósforo y del potasio sobre la producción de materia seca en morera y sus cualidades bromatológicas que permitiera hacer la respectiva comparación.

En el Cuadro 4 se mostraron los resultados experimentales de producción de biomasa y las cualidades bromatológicas de la morera influidas por el efecto conjunto o mezcla de nitrógeno, fósforo y potasio en diferentes niveles. Para la producción de biomasa se 

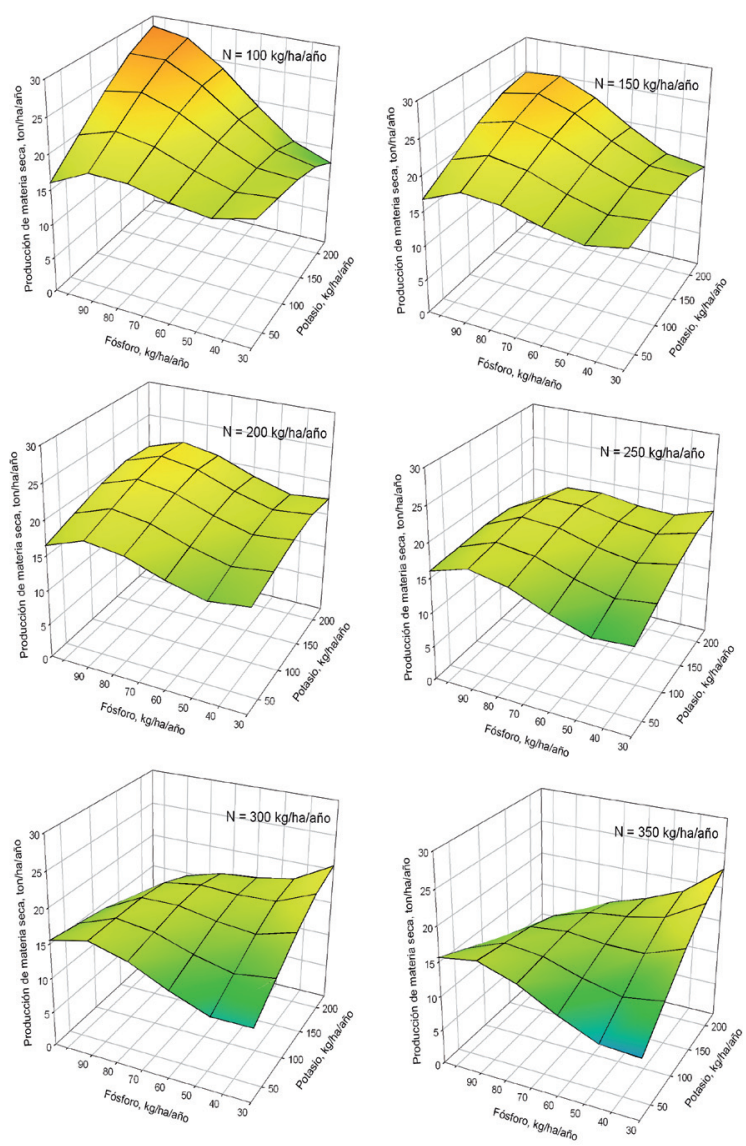

Figura 1. Respuesta de la producción de materia seca por hectárea de la morera fertilizada con diferentes cantidades de nitrógeno, fósforo y potasio. Cartago, 2007.

destaca la combinación de 112,5 kg de N/ha/año, $90 \mathrm{~kg}$ de $\mathrm{P} / \mathrm{ha} / \mathrm{año} \mathrm{y} 225 \mathrm{~kg}$ de $\mathrm{K} / \mathrm{ha} / \mathrm{año}$ con $142,7 \mathrm{t}$ de materia verde/ha/año y $28,4 \mathrm{t}$ de $\mathrm{MS} / \mathrm{ha} / \mathrm{año}$ sobre un grupo de combinaciones que alcanzaron entre 100 y $120 \mathrm{t}$ de materia verde, equivalentes entre 19 y $23 \mathrm{t}$ de $\mathrm{MS} / \mathrm{ha} /$ año. Si ésta fuese una fórmula a recomendar, vemos que la demanda de nitrógeno es relativamente mediabaja mientras que la demanda de fósforo y potasio serían relativamente media-alta. En suelos de origen volcánico, la Asociación Internacional de Industriales de Fertilizantes (IFA 1992) recomiendan $300 \mathrm{~kg}$ de N/ha/año, $160 \mathrm{~kg}$ de P/ha/año y $200 \mathrm{~kg}$ de KN/ha/año, lo cual no concuerda con los resultados expuestos. En el Cuadro 6 se observa que para obtener los máximos rendimientos de materia seca por hectárea, se requieren $240 \mathrm{~kg}$ de N/ha/año, $65 \mathrm{~kg}$ de P/ha/año y $74 \mathrm{~kg}$ de $\mathrm{K} / \mathrm{ha} / \mathrm{año}$, valores máximos obtenidos al derivar los polinomios de las superficies de respuesta correspondientes (Cuadro 5). En términos prácticos equivale a la aplicación de 24 sacos de 46 kilos (quintales)/ha/año de la fórmula 22-6-6,8 de NPK.

La composición bromatológica de la morera mostrada en el Cuadro 4, requiere un análisis desagregado entre los nutrientes que requieren ser maximizados y los que deben ser minimizados en función de los niveles de nitrógeno, fósforo y potasio requeridos para obtener los valores bromatológicos deseados en nutrición animal. Es de beneficio nutricional maximizar el contenido de materia seca y que para ello se requieren aplicar $244 \mathrm{~kg}$ de N/ha/año y $70 \mathrm{~kg}$ de P/ha/año, sin la aplicación de potasio por cuanto este elemento minimiza el contenido de materia seca (Cuadro 6) o maximiza su suculencia (el contenido de agua). Para maximizar el contenido de proteína cruda se requiere una dosis alta de potasio (146 kg/ha/año) junto con $57 \mathrm{~kg}$ de $\mathrm{P} / \mathrm{ha} / \mathrm{año}$ y $441 \mathrm{~kg}$ de N/ha/año (dosis muy alta). Se observa también que niveles altos de potasio son requeridas para maximizar la producción de fibra neutra y sus respectivos componentes, en especial el contenido de fibra ácido detergente, celulosa y lignina, lo cual es indeseable en alimentos para vacas lecheras (NRC 2001). El potasio actúa en el transporte pasivo y activo del agua dentro de la planta, en la regulación del potencial osmótico de las células vegetales, en la apertura y cierre de los estomas, en la activación de las enzimas involucradas en los procesos de fotosíntesis y respiración y en el transporte de los azúcares solubles, indispensables para el crecimiento vegetal (Taiz y Zeiger 1991, Flores-Vindas 1999).

Los resultados de la presente investigación no son concordantes con otros ensayos de fertilización efectuados bajo condiciones diferentes. Espinoza 1996, al estudiar niveles de fertilización nitrogenada (180 a $540 \mathrm{~kg} / \mathrm{ha} / \mathrm{año}$ ) en Paquera y Puriscal (Costa Rica), encontraron valores de 28 a $31 \%$ de materia seca a una altitud menor de $1.100 \mathrm{msnm}$ en rebrotes de cuatro meses. Además encontraron niveles de proteína cruda similares con un ligero incremento al aumentar el nivel de fertilizante aplicado. Esos valores se asemejan a los reportados por Rodríguez et al. (1994) al aplicar niveles de 0 a $80 \mathrm{~kg}$ de N/ha/año en el trópico seco 
Cuadro 5. Predicción de los rendimientos de materia seca y los valores bromatológicos de la morera con base en los niveles de NKP aplicados. Cartago, Costa Rica. 2007.

\begin{tabular}{|c|c|c|c|}
\hline $\begin{array}{c}\text { Componente } \\
\text { bromatológico }\end{array}$ & \multicolumn{2}{|r|}{ Ecuación de regresión } & \multirow{2}{*}{$\begin{array}{c}\mathbf{R}^{2} \\
\mathrm{R}^{2}=0,88\end{array}$} \\
\hline $\begin{array}{l}\text { Materia verde, } \\
\mathrm{kg} / \mathrm{ha} / \mathrm{año}\end{array}$ & & $\begin{array}{c}133597,0042+368,7813 \mathrm{~N}-3291,8202 \mathrm{P}-462,0533 \mathrm{~K}-2,6515 \mathrm{~N}^{2}+52,8071 \mathrm{P}^{2}+0,4288 \mathrm{~K}^{2}+0,0037 \mathrm{~N}^{3} \\
-0,2838 \mathrm{P}^{3}-0,0026 \mathrm{~K}^{3}+2,3963 \mathrm{NP}+2,6474 \mathrm{NK}+9,2278 \mathrm{PK}-0,0438 \mathrm{NPK}\end{array}$ & \\
\hline $\begin{array}{l}\text { Materia seca, } \mathrm{kg} / \\
\text { ha/año }\end{array}$ & $\mathrm{Y}=$ & $\begin{array}{c}35789,40882+29,8641 \mathrm{~N}-1009,89221 \mathrm{P}-131,32882 \mathrm{~K}-0,44786 \mathrm{~N}^{2}+15,3638 \mathrm{P}^{2}+0,17304 \mathrm{~K} 2+0,00 \\
062 \mathrm{~N}^{3}\end{array}$ & \\
\hline & & $-0,0792 \mathrm{P}^{3}-0,00078 \mathrm{~K}^{3}+0,73644 \mathrm{NP}+0,70939 \mathrm{NK}+2,28144 \mathrm{PK}-0,011 \mathrm{NPK}$ & $\mathrm{R}^{2}=0,87$ \\
\hline \multirow[t]{2}{*}{ Materia seca, $\%$} & $\mathrm{Y}=$ & $\begin{array}{c}28,78823926-0,04464241 \mathrm{~N}-0,37116854 \mathrm{P}-0,02801301 \mathrm{~K}+0,00006597 \mathrm{~N}^{2}+0,00510642 \mathrm{P}^{2}- \\
0,00000408 \mathrm{~K}^{2}-0,00000009 \mathrm{~N}^{3}\end{array}$ & \\
\hline & & $\begin{array}{c}-0,0000241 \mathrm{P}^{3}-0,00000013 \mathrm{~K}^{3}+0,00027296 \mathrm{NP}+0,00021556 \mathrm{NK}+0,00051944 \mathrm{PK}- \\
0,00000266 \mathrm{NPK}\end{array}$ & $\mathrm{R}^{2}=0,90$ \\
\hline \multirow[t]{2}{*}{$\begin{array}{l}\text { Proteína cruda, } \\
\%\end{array}$} & & $\begin{array}{c}\begin{array}{c}26,29007868-0,09727598 \mathrm{~N}+0,13479688 \mathrm{P}-0,01683615 \mathrm{~K}+0,00026476 \mathrm{~N}^{2}-0,00121667 \mathrm{P}^{2}+0,000 \\
29897 \mathrm{~K}^{2}-0,0000002 \mathrm{~N}^{3}\end{array}\end{array}$ & \\
\hline & & $+0,00000707 \mathrm{P}^{3}-0,00000068 \mathrm{~K}^{3}-0,00019963 \mathrm{NP}-0,00006 \mathrm{NK}-0,00046056 \mathrm{PK}+0,00000132 \mathrm{NPK}$ & $\mathrm{R}^{2}=0,99$ \\
\hline \multirow[t]{2}{*}{$\begin{array}{l}\text { Fibra neutro de- } \\
\text { tergente, } \%\end{array}$} & $\mathrm{Y}=$ & $\begin{array}{c}78,49458147-0,16743326 \mathrm{~N}+0,02188052 \mathrm{P}-0,15736711 \mathrm{~K}+0,00065891 \mathrm{~N}^{2}-0,00566351 \mathrm{P}^{2}+0,000 \\
90642 \mathrm{~K}^{2}-0,00000105 \mathrm{~N}^{3}\end{array}$ & \\
\hline & & $\begin{array}{c}+0,0000397 \mathrm{P}^{3}-0,00000279 \mathrm{~K}^{3}+0,00065741 \mathrm{NP}+0,00024311 \mathrm{NK}+0,00137833 \mathrm{PK}- \\
0,0000391 \mathrm{NPK}\end{array}$ & $\mathrm{R}^{2}=0,99$ \\
\hline \multirow[t]{2}{*}{$\begin{array}{l}\text { Fibra ácida deter- } \\
\text { gente, } \%\end{array}$} & $\mathrm{Y}=$ & $\begin{array}{c}39,59725081-0,02015848 \mathrm{~N}-0,00880058 \mathrm{P}+0,078191 \mathrm{~K}+0,00007385 \mathrm{~N}^{2}-0,00187966 \mathrm{P}^{2}- \\
0,00116502 \mathrm{~K}^{2}-0,00000022 \mathrm{~N}^{3}\end{array}$ & \\
\hline & & $\begin{array}{c}+0,00000582 \mathrm{P}^{3}+0,00000246 \mathrm{~K}^{3}+0,00037741 \mathrm{NP}+0,00016815 \mathrm{NK}+0,00098389 \mathrm{PK}- \\
0,00000207 \mathrm{NPK}\end{array}$ & $\mathrm{R}^{2}=0,96$ \\
\hline \multirow[t]{2}{*}{ Hemicelulosa, \% } & $Y=$ & $\begin{array}{c}38,86549665-0,14696414 \mathrm{~N}+0,03184621 \mathrm{P}-0,23580303 \mathrm{~K}+0,00058405 \mathrm{~N}^{2}- \\
0,00379804 \mathrm{P}^{2}+0,0020741 \mathrm{~K}^{2}-0,00000083 \mathrm{~N}^{3}\end{array}$ & \\
\hline & & $\begin{array}{c}+0,00003395 \mathrm{P}^{3}-0,00000526 \mathrm{~K}^{3}+0,00027852 \mathrm{NP}+0,00007467 \mathrm{NK}+0,00039333 \mathrm{PK}- \\
0,00000183 \mathrm{NPK}\end{array}$ & $\mathrm{R}^{2}=0,98$ \\
\hline \multirow[t]{2}{*}{ Celulosa, $\%$} & $\mathrm{Y}=$ & $\begin{array}{c}40,2305919-0,11723326 \mathrm{~N}-0,15211112 \mathrm{P}-0,06050729 \mathrm{~K}+0,00036033 \mathrm{~N}^{2}-0,00003565 \mathrm{P}^{2}- \\
0,00061034 \mathrm{~K}^{2}-0,00000051 \mathrm{~N}^{3}\end{array}$ & \\
\hline & & $\begin{array}{c}-0,00000195 \mathrm{P}^{3}+0,00000124 \mathrm{~K}^{3}+0,00029222 \mathrm{NP}+0,00035333 \mathrm{NK}+0,00159278 \mathrm{PK}- \\
0,00000302 \mathrm{NPK}\end{array}$ & $\mathrm{R}^{2}=0,90$ \\
\hline \multirow[t]{2}{*}{ Lignina, \% } & $\mathrm{Y}=$ & $\begin{array}{c}-0,5827296657+0,0968659143 \mathrm{~N}+0,1419424589 \mathrm{P}+0,1384855367 \mathrm{~K}-0,0002862135 \mathrm{~N}^{2}- \\
0,001831146 \mathrm{P}^{2}-0,0005555355 \mathrm{~K}^{2}+0,0000002916 \mathrm{~N}^{3}\end{array}$ & \\
\hline & & $\begin{array}{c}+0,0000077175 \mathrm{P}^{3}+0,000001224 \mathrm{~K}^{3}+0,0000874074 \mathrm{NP}-0,0001842963 \mathrm{NK}-0,0006044444 \mathrm{PK}+ \\
0,0000009284 \mathrm{NPK}\end{array}$ & $\mathrm{R}^{2}=0,94$ \\
\hline \multirow[t]{2}{*}{$\begin{array}{l}\text { Extracto etéreo, } \\
\%\end{array}$} & $\mathrm{Y}=$ & $\begin{array}{c}1,636945989-0,006534404 \mathrm{~N}-0,016325413 \mathrm{P}+0,026048841 \mathrm{~K}+0,000036305 \mathrm{~N}^{2}+0,000154728 \mathrm{P}^{2}- \\
0,00014334 \mathrm{~K}^{2}-0,000000051 \mathrm{~N}^{3}\end{array}$ & \\
\hline & & $\begin{array}{c}-0,000001 \mathrm{P}^{3}+0,000000256 \mathrm{~K}^{3}+0,000026296 \mathrm{NP}-0,000019704 \mathrm{NK}-0,000010556 \mathrm{PK}+0,0000002 \\
12 \mathrm{NPK}\end{array}$ & $\mathrm{R}^{2}=0,80$ \\
\hline \multirow[t]{2}{*}{ Cenizas, $\%$} & $\mathrm{Y}=$ & $\begin{array}{c}11,6777466+0,02781462 \mathrm{~N}+0,13702555 \mathrm{P}-0,00008181 \mathrm{~K}-0,00007609 \mathrm{~N}^{2}-0,0028432 \mathrm{P}^{2}-0,00000 \\
989 \mathrm{~K}^{2}+0,00000008 \mathrm{~N}^{3}\end{array}$ & \\
\hline & & $\begin{array}{c}+0,00001455 \mathrm{P}^{3}+0,00000014 \mathrm{~K}^{3}+0,00005741 \mathrm{NP}-0,00007956 \mathrm{NK}-0,00004389 \mathrm{PK}+0,00000076 \\
\text { NPK }\end{array}$ & $\mathrm{R}^{2}=0,76$ \\
\hline
\end{tabular}

de Guatemala. Resultados similares hallaron Rojas y Benavides (1994) y Araya et al. (1994). Todos estos resultados obtenidos en experimentos realizados en Centroamérica son similares a los encontrados por
Bangash y Sheikh (1990) en Pakistán al estudiar diferentes combinaciones de NPK sobre la producción de forrajes y la calidad nutricional. Los resultados encontrados por Dávila et al. (2004) en Venezuela, al 
Cuadro 6. Requerimientos óptimos de NPK para la producción de materia seca y su composición bromatológica en morera. Cartago, Costa Rica. 2007.

\begin{tabular}{lccc}
\hline \multicolumn{4}{c}{ Elementos nutricionales } \\
& $\begin{array}{c}\text { N, kg/ha/ } \\
\text { año }\end{array}$ & $\begin{array}{c}\text { P, kg/ha/ } \\
\text { año }\end{array}$ & $\begin{array}{c}\text { K, kg/ha/ } \\
\text { año }\end{array}$ \\
\hline $\begin{array}{l}\text { Materia verde, t/ha/ } \\
\text { año }\end{array}$ & 238,87 & 62,02 & 54,97 \\
Materia seca, t/ha/año & 240,78 & 64,66 & 73,95 \\
Materia seca, \% & 244,33 & 70,63 & $-10,46$ \\
Proteína cruda, \% & 441,27 & 57,36 & 146,55 \\
Fibra neutro detergen- & & & \\
te, \% & 209,18 & 47,55 & 108,29 \\
Fibra ácida detergen- & & & \\
te, \% & 111,89 & 107,66 & 157,86 \\
Hemicelulosa, \% & 234,56 & 37,29 & 131,44 \\
Celulosa, \% & 235,51 & $-6,09$ & 164,07 \\
Lignina, \% & 327,18 & 79,09 & 151,29 \\
Extracto etéreo, \% & 237,29 & 51,58 & 186,64 \\
Cenizas, \% & 317,04 & 65,14 & 23,55 \\
Requerimientos & & & 117,69 \\
promedio & 262,03 & 56,69 & \\
\hline
\end{tabular}

estudiar el efecto de la fertilización NPK en la producción de forraje de la asociación kikuyo-maní forrajero, tienen la misma tendencia a la observada en este experimento con morera, guardando las diferencias en la magnitud de la respuesta entre especies distintas y de porte totalmente diferente.

\section{CONCLUSIONES Y RECOMENDACIONES}

Los niveles de nitrógeno, fósforo y potasio mostraron variaciones importantes como efecto principal sobre los parámetros de producción de biomasa, tanto verde como seca, y sobre los valores bromatológicos de la morera.

La mezcla de las diferentes fuentes estudiadas que mejor reflejan el comportamiento promedio de óptimos en producción y contenido bromatológico es un requerimiento de $262 \mathrm{~kg} / \mathrm{ha} /$ año nitrógeno, 56,7 $\mathrm{kg} / \mathrm{ha}$ /año de fósforo y $117,7 \mathrm{~kg} / \mathrm{ha}$ /año de potasio, equivalente a una proporción de 4,6:1:2 de NPK.

El requerimiento indicado es similar al demandado para lograr la máxima producción de materia seca por unidad de área, pero para optimizar la concentración de proteína cruda es necesario agregar una aplicación de nitrógeno en $180 \mathrm{~kg} / \mathrm{ha} / \mathrm{año}$, manteniendo el nivel de fósforo y potasio descrito previamente. Es evidente que desde el punto de vista nutricional del animal, no se desea maximizar la producción de fibra neutra detergente, cuya demanda para ello se encuentra en 209 $\mathrm{kg}$ de N/ha/año, $47 \mathrm{~kg}$ de P/ha/año y $108 \mathrm{~kg}$ de K/ha/ año, equivalente a disminuir $53 \mathrm{~kg}$ de N/ha/año de la aplicación para maximizar la producción de forraje.

En el presente experimento se usó un diseño para explorar el efecto de diferentes niveles de nitrógeno, fósforo y potasio requeridos para maximizar la producción de materia seca y sus componentes bromatológicos. Se recomienda revalidar los resultados obtenidos mediante un factorial completo empleando niveles de nitrógeno entre 150 y $350 \mathrm{~kg} / \mathrm{ha} / \mathrm{año}$, de fósforo entre 40 y $80 \mathrm{~kg} / \mathrm{ha} / \mathrm{año} \mathrm{y} \mathrm{de} \mathrm{potasio} \mathrm{entre} 50$ y $175 \mathrm{~kg} / \mathrm{ha} / \mathrm{año}$, buscando maximizar la producción de materia seca por hectárea y el contenido de proteína cruda, y a la vez minimizando el contenido de fibra detergente y sus componentes.

\section{LITERATURA CITADA}

AOAC. 2002. Association of Official Analysis Chemistry. Animal Feeds. Washington, D.C. USA. Chapter 4. p. $1-6$.

Araya, J; Benavides, JE; Arias, R; Ruiz, A. 1994. Identificación y caracterización de árboles y arbustos con potencial forrajero. In: Árboles y arbustos forrajeros en América Central. Turrialba, Costa Rica. CATIE, Volumen 1 p. 31-63.

Bangash, SH; Sheikh, MI. 1990. Effect of NPK fertilizer on foliage yield and nutritive value of mulberry (Japanese source). Pakistan Journal of Forestry 30:137-138.

Benavides, J. 1981. Efecto de diferentes niveles de suplementación con follaje de Morera (Morus sp) sobre el crecimiento y consumo de corderos alimentados con pasto (Pennisetum purpureum). Proyecto CATIE-ROCAP. Turrialba. Costa Rica. Boletín Técnico \#7. p. 40-42.

Benavides, J; Borel, R; Esnaola, MA. 1986. Evaluación de la producción de forraje del árbol de morera (Morus 
sp) sometido a diferentes frecuencias y altura de corte. Inst. Centro Agronómico Tropical de Investigación y Enseñanza. Resumen de las investigaciones realizadas con rumiantes menores, en el Proyecto de Sistemas de Producción Animal. Turrialba, Costa Rica. Serie Técnica. Informe Técnico No 68. p. 74-76.

Benavides, J; Lachaux, M; Fuentes, M. 1994. Efecto de la aplicación de estiércol de cabra en el suelo sobre la calidad y producción de biomasa de Morera (Morus $s p$ ). Arboles y Arbustos Forrajeros en América Central. Turrialba, Costa Rica. Serie Técnica. CATIE. Informe Técnico No 236. Volumen II. p. 495-514.

Blanco, R. 1992. Distancia de siembra y altura de corte en la producción y calidad del forraje de Morera (Morus $s p$ ) en el parcelamiento Cuyunta, Escuintla Guatemala. Guatemala. Universidad de San Carlos. 15 p.

Boschini, C; Dormond, H; Castro, A. 1998. Producción de biomasa de la morera (Morus alba) en la Meseta Central de Costa Rica, establecida y cosechada a diferentes distancias de siembra, alturas y frecuencias de poda. Revista Agronomía Mesoamericana 9: 28-39.

Boschini, C; Dormond, H; Castro, A. 1999. Respuesta de la morera (Morus alba) a la fertilización nitrogenada, densidades de siembra y a la defoliación. Revista Agronomía Mesoamericana 10:7-16.

Boschini, C. 2002. Establishment and management of mulberry for intensive forage production. Mulberry for animal production. FAO, Roma. Animal Production and Health Paper 147. p. 115-122.

Boschini, C. 2008. Diseños experimentales especiales en la investigación con ganado lechero. Serie Agrotecnológica, Volumen 6. Editorial Universidad de Costa Rica. 116 p.

Castro, A. 1989. Producción de leche de cabras alimentadas con King grass (Pennisetum purpureum x $P$. typloides), suplementados con diferentes niveles de follaje de Poró (E. poeppigrama) y de fruto de plátano (Musa sp. var. Pelipita). Tesis M.Sc. Turrialba, C.R. UCR/CATIE. 58 p.

Cochran, WG; Cox, GM. 1957. Experimental designs. Second Edition. John Wiley \& Sons. New York. 611 p.

Dávila, C; Castro, F; Urbano, D. 2004. Efecto de la presión de pastoreo y fertilización NPK en la producción de forraje de la asociación kikuyo-maní forrajero en el estado Mérida. Zootecnia Tropical 22:157-166.

Elizondo, JA. 2007. Producción y calidad de la biomasa de morera (Morus alba) fertilizada con diferentes abonos. Agronomía Mesoamericana 18:255-261.
Espinoza, E. 1996. Efecto del sitio y del nivel de fertilización nitrogenada sobre la producción y calidad de tres variedades de morera en Costa Rica. Tesis de Maestría. CATIE. Turrialba, Costa Rica. 72 p.

Estrada, A. 1995. Efecto de la sustitución de king grass por morera sobre los parámetros de degradación y fermentación ruminal de cuatro forrajes de calidad constante. Tesis de Maestría. CATIE. Turrialba, Costa Rica. 63 p.

Flores-Vindas, E. 1999. La planta estructura y función. Volumen 1. Editorial Libro Universitario Regional (LUR). Costa Rica. 367 p.

Goering, HK; Van Soest, PJ. 1970. Forage fiber analysis (Apparatus, reagents, procedures and some applications). Agricultural Handbook No. 379. ARS-USDA, Washington, DC. p. 76.

González, J. 1996. Evaluación de la calidad nutricional de la morera fresca y ensilada con bovinos de engorde. Tesis de Maestría. CATIE. Turrialba, Costa Rica. 67 p.

IFA (International Fertilizer Industry Association). 1992. IFA World Fertilizer Use Manual. Mulberry Chart. BASF. Aktiengesells- Chaft. Agricultural Research Station. Germany. p. 595-601.

NRC (National Research Council). 2001. Nutrient requirements of dairy cattle. Seventh Revised Edition. National Academy Press. Washington, USA. 381 p.

Paolieri, L. 1970. Competicao de variedades de Amoreiras. Boletín Técnico de Sericultura No 57. Secretaría de Agricultura. Coordinadora de Pesquiza Agropecuaria. Instituto de Zootecnia. Secao de Sericultura. Brasil. p. 3-16.

Rodríguez, C; Arias, R; Quiñones, J. 1994. Efecto de la frecuencia de poda y el nivel de fertilización nitrogenada, sobre el rendimiento y calidad de la biomasa de Morera (Morus sp) en el trópico seco de Guatemala. Arboles y arbustos forrajeros en América Central. Turrialba, Costa Rica. Serie Técnica. CATIE. Informe Técnico No 236. Volumen II. p. 515-528.

Rojas, H; Benavides, JE. 1994. Producción de leche en cabras alimentadas con pasto suplemento con altos niveles de Morera (Morus alba). In: Árboles y arbustos forrajeros en América Central. Turrialba, Costa Rica. CATIE, Volumen 1. p. 305-320.

SAS. 1985. Statistical Analysis System. SAS User's Guide: Statistics (Version 5 Ed.) SAS Institute Inc. Cary, NC. USA. 956 p.

Taiz, L; Zeiger, E. 1991. Plant physiology. The Benjamin/ Cummings Publishing Company, Inc. California, USA. 565 p. 
Ting-Zing, Z; Yun-Fan, T; Guang-Xien, H; Huaizhong, F; Ben, MA. 1988. Mulberry cultivation. FAO Agricultural Services Bulletin 73/1. Food and Agricultural Organization of the United Nations. Rome. 127 p.

Vásquez, A. 1982. Estudio detallado de los suelos de la Estación Experimental de Ganado Lechero El Alto.
Escuela de Fitotecnia, Facultad de Agronomía, Universidad de Costa Rica. 36 p.

Velázquez, CM. 1992. El forraje de morera (Morus sp) como fuente de proteína en dietas a base de ensilado de sorgo (Sorghum bicolor x Sorghum sudanense) para novillos en el parcelamiento Cuyutá. Tesis de Licenciatura. Universidad de San Carlos, Guatemala. 43 p. 